\title{
ARTICLE
}

\section{Benthic food distribution as a predictor of the spatial distribution for shorebirds in a wetland of central Chile}

La distribución del alimento bentónico como predictor de la distribución espacial de playeros en un humedal de Chile central

\section{Alfredo D. Pérez-Vargas ${ }^{1,2,3}$, Mariano Bernal ${ }^{1}$, Camila S. Delgadillo ${ }^{1}$, Eduardo F. González-Navarro and Mauricio F. Landaeta ${ }^{4}$}

${ }^{1}$ Facultad de Ciencias del Mar y de Recursos Naturales, Universidad de Valparaíso, Avenida Borgoño 16344, Reñaca, Viña del Mar, Chile. alfredo.perez@uv.cl

${ }^{2}$ Programa de Doctorado en Ciencias mención Recursos Naturales Acuáticos, Facultad de Ciencias del Mar y de Recursos Naturales, Universidad de Valparaíso, Avenida Borgoño 16344, Reñaca, Viña del Mar, Chile

${ }^{3}$ Programa de Magíster en Oceanografía, Facultad de Ciencias del Mar y de Recursos Naturales, Universidad de Valparaíso (Programa conjunto con la Pontificia Universidad Católica de Valparaíso), Avenida Borgoño 16344, Reñaca, Viña del Mar, Chile

${ }^{4}$ Laboratorio de Ictioplancton (LABITI), Facultad de Ciencias del Mar y de Recursos Naturales, Universidad de Valparaíso, Avenida Borgoño 16344, Reñaca, Viña del Mar, Chile

Resumen.- Los ambientes costeros de Chile central son sitios importantes para playeros residentes y migratorios. Los ambientes estuarinos son hábitats críticos que proveen sitios de descanso y recursos alimentarios para playeros que recuperan energía durante su migración. Se hipotetizó que los playeros escogen hábitats específicos en el humedal del Río Aconcagua, Chile central, basados en la disponibilidad de alimento. Para probar esta hipótesis se evaluó la composición, abundancia y distribución espacial del ensamble bentónico en áreas de alimentación. La predicción principal fue que el tipo de alimento reflejaría la distribución espacial de playeros. El total de invertebrados bentónicos fue 11.514 individuos, correspondiente a 25 taxa, siendo crustáceos, poliquetos, oligoquetos e insectos los grupos taxonómicos mayores. El total de playeros fue 2.105 , y 1.136 individuos en actividad alimentaria correspondiente a 18 especies de las Familias Charadriidae, Haematopodidae, Recurvirostridae y Scolopacidae. Las planicies intermareales del área de estudio exhiben distribuciones espaciales no aleatorias de playeros en actividad alimentaria, relacionadas a la disponibilidad de alimento al interior del humedal del Río Aconcagua. El análisis integrado mostró diferencias significativas entre planicies intermareales basadas en la abundancia y composición del ensamble bentónico (mediado por las características del sedimento), y también algunas relaciones entre playeros en actividad alimentaria y grupos tróficos en planicies intermareales particulares. Estos resultados tienen implicancias para la conservación de playeros y la gestión de humedales, principalmente en relación a cambios ambientales, pérdida y destrucción de hábitat relacionados con la declinación de poblaciones de playeros a nivel mundial.

Palabras clave: Playeros, Charadriiformes, presa, invertebrados, humedal, Río Aconcagua, Chile central

Abstract.- Coastal landscapes of central Chile are important places for species of resident and migratory shorebirds. Estuarine environments are critical habitats that provide resting places and food resources for large concentrations of shorebirds to replenish their energy during their migration. We hypothesized that shorebirds choose particular habitats based on food availability in a tidal wetland of Aconcagua River, central Chile. We evaluated composition and abundance of benthic ensemble in foraging areas, and its local spatial distributions in these foraging areas. The main prediction was that selected trophic items may reflect the main distribution of shorebirds. The total benthic invertebrates registered were 11,514 individuals, corresponding to 25 taxa, being crustaceans, polychaetes, oligochaetes, and insects the major taxonomic groups. The total and foraging shorebirds registered were 2,105 and 1,136 individuals, respectively; corresponding to 18 species belonged to 4 Families: Charadriidae, Haematopodidae, Recurvirostridae and Scolopacidae. Tidal flats around the study site exhibit non-random spatial distributions of feeding shorebirds, responding to food availability within the Aconcagua river wetland. Our integrated analysis showed significant differences between tidal flat areas based on abundance and composition of benthic trophic items (mediated by sediment characteristics), and some relationships between foraging shorebirds and trophic items in particular tidal flat areas. These results have implications for shorebird conservation and future wetland management, mainly in relation to environmental changes and other causes of habitat loss and destruction linked with worldwide decline in many shorebird populations.

Key words: Shorebirds, Charadriiformes, prey, invertebrates, tidal wetland, Aconcagua River, central Chile 


\section{INTRODUCTION}

Coastal landscapes of central Chile are important places for at least 27 species of resident and migratory shorebirds (Schlatter \& Sielfeld 2006). During the austral winter and the spring season, these environments include austral species travelling northward and boreal long distance migratory species during southward migration to temperate grounds respectively (Morrison \& Myers 1987, Sallaberry et al. 1996, Aparicio 2006).

Estuarine environments with diverse amount of available tidal flats are critical habitats that provide resting places and food resources for large concentrations of shorebirds that use them as wintering staging areas where they need to stay for long periods or just few days as stopover sites to replenish their energy (Piersma 1987, Skagen 2006, Warnock 2010) during their south and northward migration (Myers et al. 1987, Morrison \& Ross 1989).

Shorebirds detect prey by visual and tactile sensory mechanisms, been pecking and probing the main methods (Baker \& Baker 1973), and flexible foraging strategies that can shift their diet to exploit locally abundant invertebrate resources (Skagen \& Oman 1996, Quinn \& Hamilton 2012). During nonbreeding seasons many shorebirds often forage across flats following tidal exposure and generally distribute themselves on specific habitats based on availability, distribution and abundance of macrobenthic invertebrates (Wilson 1990, Colwell \& Landrum 1993, Butler et al. 2001), according to the physical characteristics of tidal flats (Yates et al. 1993, Colwell 1993, Finn et al. 2008). Then, they move to supratidal areas to rest when the tide rises (Burger et al. 1977).

Despite the abundant literature for the Northern Hemisphere and the recent studies in South America, the shorebird usage of coastal habitats and prey resources has been scarcely studied in Chile (Tabilo et al. 1996, Aparicio 2006, Estades et al. 2009, González et al. 2011). Along Chilean coast, these environments are discontinuous and small in size $(<10 \mathrm{ha})$, and their average number and extension increase in a north-south direction (Marquet et al. 2012), being important for shorebirds during their migration. The coastal wetlands of central Chile are characterized by smooth gradients both in terms of physical properties, such as sediment grain size distributions, and biological properties, such as density of meio- and macrozoobenthic species (Kraan et al. 2009, Ge et al. 2011, Suazo et al. 2012). Oligochaetes, polychaetes, ostracods, amphipods, and chironomids are the main taxa found in these environments (Jaramillo et al. 1985, Richter 1985, Quijón \& Jaramillo 1993, Torres et al. 2012).
We hypothesized that shorebirds choose particular habitats based, at least, on food availability. The goal of this study is to test that food availability is a predictor of the spatial distributions of shorebirds species in a tidal flat from central Chile. To examine how food availability determines the spatial distributions of shorebirds, specifically we evaluated (1) composition (richness) and abundance of meio- and macrobenthic trophic items in foraging areas, and (2) its local spatial distributions in these foraging areas. The main prediction is that selected trophic items may reflect the main distribution of shorebirds. The hypothesis was tested during the austral winter and spring season in the wetlands of Aconcagua River, central Chile.

\section{MATERIALS AND METHODS}

\section{STUdy SITE}

The study site was located at wetlands of Aconcagua River (32 55'03”'S, 71³0’20”'W) (Fig. 1), a microtidal estuary (1.7 m tidal average) in central Chile (Martínez \& Cortez 2007). The wetland of Aconcagua River is shallow and contains narrow soft-sediment flats that are exposed during low tide on a semidiurnal basis. The tidal flats are utilized by shorebirds for both resting and feeding. We established 5 intertidal sand flat areas (ca. $285 \mathrm{~m}$ length, $2 \mathrm{~m}$ width each) around the lagoon formed at wetland of Aconcagua River. 'Playa Sur (PS)' correspond to a sand flat located immediately south of river mouth. 'Playa Norte (PN)' correspond to a sand flat area located immediately north of river mouth. 'Brazo Norte (BN)' was the most sheltered of the group; it was bordered on the landward side by emergent vegetation at the northeast margin of the lagoon. 'Totoral $\mathrm{La}$ Isla (TLI)' located front of river mouth and bordered on the landward side by emergent vegetation. 'Totoral Sur (TS)' located at the south margin of the lagoon, and bordered on the landward side by emergent vegetation.

\section{SHOREBIRDS SAMPLING}

Shorebird surveys were conducted on 12 dates, as sampling units, between July and December 2013 by 2 observers. In order to support statistical analysis we used additional 10 dates between August and December 2012 utilizing the same methodology as explained above. During the present study we considered only foraging shorebirds occurring on intertidal flats or wadding in the near shore of each flat. Shorebird surveys, made on days without rainfall, and with good conditions of visibility were conducted by point count at $3-\mathrm{m}$ height from 2 


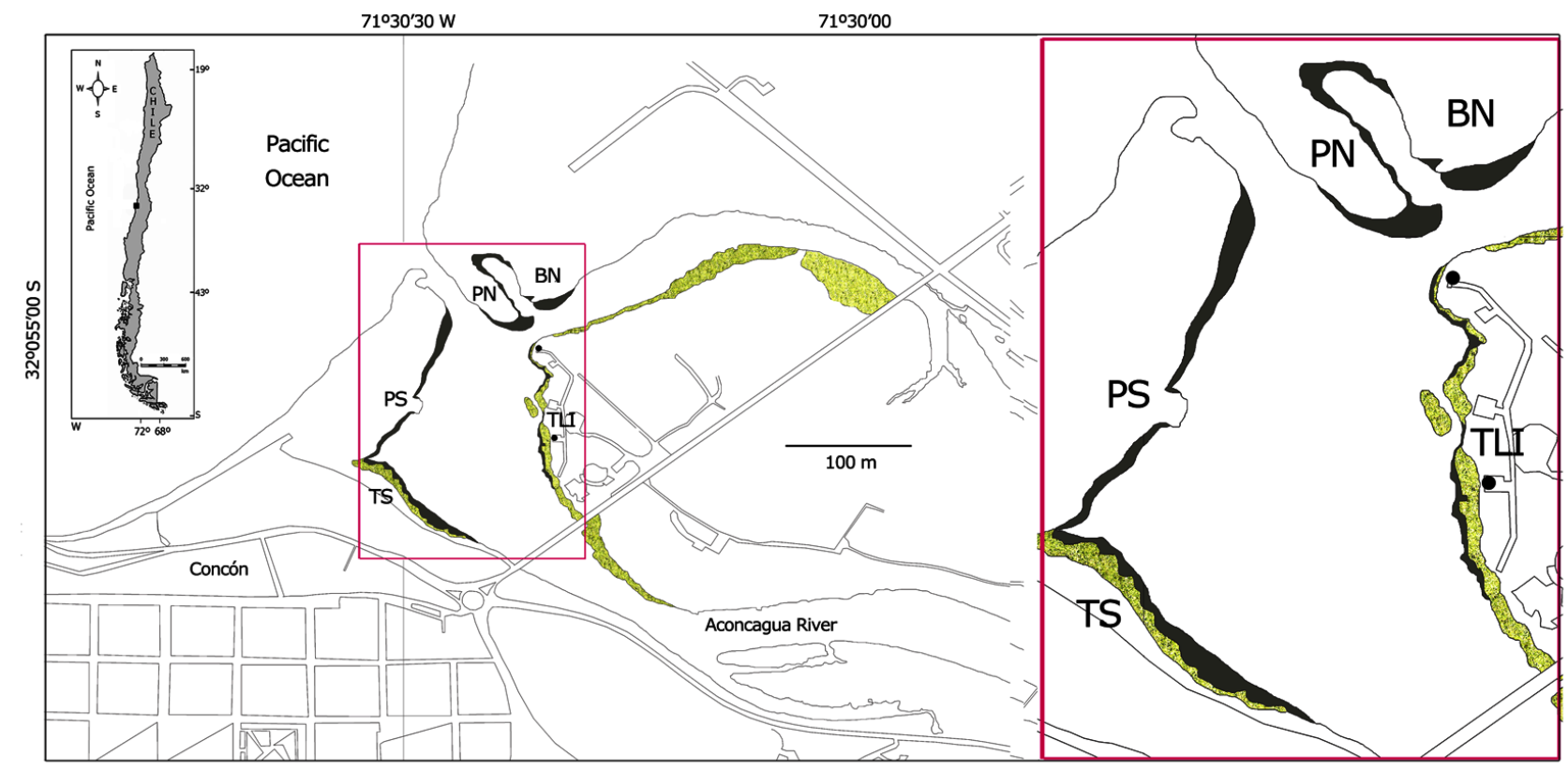

Figure 1. M ap showing the study site at wetlands of Aconcagua River and intertidal sand flat areas (PS: Playa Sur; PN: Playa Norte; BN: Brazo Norte; TL: Totoral La Isla; TS: Totoral Sur). Gray depict main tidal flats appearing at low tides. Green depict emergent vegetation. Black dots depict bird point counts / Sitio de estudio en el humedal del Río Aconcagua y planicies intermareales (PS: Playa Sur; PN: Playa Norte; BN: Brazo Norte; TLI: Totoral La Isla; TS: Totoral Sur). Gris denota las planicies intermareales que emergen en baja marea. Verde denota la vegetación emergente. Puntos negros indican los puntos de observación y conteo de aves

vantage points (Bibby et al. 2000) over 60-min intervals beginning, as possible, one hour before low tide, and ending one hour after low tide. Because every flat was fully visible from these vantage points, double-counting of birds moving between flats was avoided. If a bird did move between flat areas, its prior location was the one recorded. Observations and identification were made using 7x50 binoculars, 60x spotting scope and tally counter for counting birds at a minimum distance of $\sim 20 \mathrm{~m}$ from foraging birds. This distance was sufficient to avoid disturbing the birds.

\section{BENTHOS SAMPLING}

We determined composition and abundance of meio- and macrozoobenthos in the wetland of Aconcagua River between July and December 2013. Benthos sampling was performed monthly over 5 intertidal sand flat areas. The sampling stations, located on the water line of each flat, were visited by foot during low tide on next day of the shorebird survey. Three sample units were collected at each area. Each sample unit consisted of sediment taken down to a depth of $10 \mathrm{~cm}$ with a core of $0.002 \mathrm{~m}^{2}$. The $10-\mathrm{cm}$ depth was selected to capture benthic organisms related to longest bill range of probing shorebirds (Johnson et al. 2007, VanDusen et al. 2012). In order to obtain a general composition of benthic ensemble the sample units were not sieved. The 90 sample units were carried to the laboratory on ice with contents fixed using $4 \%$ diluted formalin with distilled water. After sorting and enumerating by taxonomic groups, organisms were preserved in $80 \%$ diluted ethanol with distilled water.

\section{Sediments SAMPLing}

Sediment sample units were collected concurrently with benthic organisms. Three sample units were collected at each station. Each sample unit consisted of sediment taken down to a depth of $10 \mathrm{~cm}$ with a $5-\mathrm{cm}$ diameter core. All sediment sample units were taken at low tide. Each sediment sample unit was dried for $48 \mathrm{~h}$ at $70^{\circ} \mathrm{C}$ in the laboratory and then weighed and sieved through 7 meshes. Grain sizes were binned into 7 groups based on the Udden-Wentworth scale (Udden 1914, Wentworth 1922) (silt/clay: $<0.063 \mathrm{~mm}$, very fine sand: $<0.125 \mathrm{~mm}$, fine sand: $<0.250 \mathrm{~mm}$, medium sand: $<0.500 \mathrm{~mm}$, coarse sand: $<$ $1.000 \mathrm{~mm}$, very coarse sand: $<2.000 \mathrm{~mm}$, gravel: $\geq 2000 \mathrm{~mm}$ ), and percent composition was computed for each sample unit using GRADISTAT software package v4 (Blott \& Pye 2001).

\section{Data analysis}

In order to consider seasonal variability of biological matrices, the database was analyzed separately as austral winter and spring periods. Very small size benthic taxa $(<0.4 \mathrm{~cm})$ were 
excluded for all analysis. Response variables (i.e., benthos abundance, shorebirds abundance, and grain size of sediments) were $\log (\mathrm{x}+1)$-transformed to prove statistical assumptions of normality (Lilliefors test) and homogeneity of variance (Levene test) (STATISTICA 7.0) ${ }^{1}$. In all cases, it was not possible to reach the assumptions for parametric statistical procedures. To determine whether benthic and shorebirds abundances differed among flats, we performed Kruskal-Wallis test, with flat area as the independent variable and individual sampling units as replicates. To test for among-flat differences in grain-size group means, we performed Kruskal-Wallis test, with flat area as the independent variable and individual sampling units as replicates (STATISTICA 7.0). Significant differences were followed by Mann-Whitney test with Bonferroni correction test a posteriori contrasts to compare benthic abundance, foraging shorebirds abundance, and the grain-size group means distribution at each tidal flat (PAST 2.17c, Hammer et al. 2001).

To determine whether shorebirds ensemble composition differed among flats, we performed one-way ANOSIM based on Bray-Curtis similarity matrix computed on abundance $(\log (\mathrm{x}+1)$ transformed), with flat area as the independent variable and individual sampling units as replicates. Todetermine whether benthic community composition differed among flats, we performed one-way ANOSIM based on Bray-Curtis similarity matrix computed on abundance $(\log (x+1)$ transformed $)$, with flat area as the independent variable and individual sampling units as replicates. To test for among-flat differences in grainsize distribution, we performed a one-way ANOSIM based on a Euclidean distance resemblance matrix with flat area as the independent variable and grain-size distributions from individual sampling units as replicates.

The relationship between benthic community structure and the shorebird ensemble was assessed using the BEST procedure (PRIMER-E 6.1.6, Clarke \& Gorley 2006). BEST searches for high rank correlations between a fixed similarity matrix and resemblance matrices produced from a subset of possible explanatory variables that come from a second 'active' similarity matrix. The degree to which the multivariate patterns of the fixed matrix match the patterns of the optimized subset matrix is the degree to which the subset variables 'explain' the patterns in the fixed matrix. This analysis was performed using the shorebird and benthic abundances datasets, with the similarity matrix from the shorebird dataset serving as the fixed matrix. We performed a BEST analysis using the fixed shorebird BrayCurtis similarity matrix and the active benthic Bray-Curtis similarity matrix from the reduced species set to major taxonomic groups. Our fixed matrix was the Bray-Curtis similarity matrix produced from the shorebird ensemble dataset previously $\log (\mathrm{x}+1)$-transformed. The BEST procedure was used to assess the relationship between sediment grain-size composition and the shorebird ensemble. The shorebird abundance data were $\log (\mathrm{x}+1)$-transformed, and their BrayCurtis similarity matrix served as the fixed matrix for the BEST analysis. Both benthic and sediment datasets were reduced by averaging sampling units. A final BEST analysis drew upon all three datasets. The composited sediment and benthic macrofauna datasets were combined on a single spreadsheet to form an active matrix that supplied explanatory variables from both datasets at the same time. Again, the Bray-Curtis similarity matrix from the shorebird dataset served as the fixed matrix for the BEST analysis.

To determine spatial distribution of foraging shorebird species we examined the relationship between $\bar{x}$ abundance and $s^{2}$ in each intertidal flat area. Finally, in order to show which foraging shorebird species, and benthic food items, are associated with which particular tidal flat area, we performed the chi-square contingency table analysis.

\section{ReSUltS}

\section{ShOREBIRDS ANALYSIS}

The total and the foraging shorebirds registered were 2,105 and 1,136 individuals, respectively, corresponding to 18 species belonging to 4 Families: Charadriidae, Haematopodidae, Recurvirostridae and Scolopacidae. Species recorded were 4 residents, 12 boreal migrants and 2 austral migrants.

The more common and abundant foraging shorebirds were the resident black-necked stilt Himantopus mexicanus (Recurvirostridae) (27\%), southern lapwing Vanellus chilensis (Charadriidae) (21\%), american oystercatcher Haematopus palliatus (Haematopodidae) (5\%) and the migrants: greater yellowlegs Tringa melanoleuca (13\%) and whimbrel Numenius phaeopus (Scolopacidae) (10\%). Other shorebirds occurred irregularly: golden plover Pluvialis dominica, blackbellied plover Pluvialis squatarola, semipalmated plover Charadrius semipalmatus, ruddy turnstone Arenaria interpres, sanderling Calidris alba, Baird's sandpiper Calidris bairdii, pectoral sandpiper Calidris melanotos, hudsonian godwit Limosa haemastica, willet Tringa semipalmata, lesser yellowlegs Tringa flavipes, snowy plover Charadrius nivosus, collared plover Charadrius collaris and rufous-chested dotterel Charadius modestus (Table 1).
${ }^{1}$ StatSoft Inc. 2004. STATISTICA (data analysis software system), version 7 . 
Table 1. Abundance ( $\operatorname{mean} \pm S D)$ of foragingshorebirds registered by sandflat areaduringaustral winter and springseasons, PS: Playa Sur; PN: PlayaNorte; BN: Brazo Norte; TU: Totoral La Isla; TS: Totoral Sur / Abundancia (media \pm DE) de aves playeras alimentándose registradas por planicie costera durante las estaciones de invierno y primavera. PS: Playa Sur; PN: Playa Norte; BN: Brazo Norte; TLI: Totoral La Isla; TS: Totoral Sur

\begin{tabular}{|c|c|c|c|c|c|c|c|c|c|c|}
\hline & \multicolumn{5}{|c|}{ AUSTRAL WINTER } & \multicolumn{5}{|c|}{ AUSTRAL SPRING } \\
\hline & PS & $\mathrm{PN}$ & $\mathrm{BN}$ & TLI & TS & PS & $\mathrm{PN}$ & $\mathrm{BN}$ & TLI & TS \\
\hline & Mean (SD) & Mean (SD) & Mean (SD) & Mean (SD) & Mean (SD) & Mean (SD) & Mean (SD) & Mean (SD) & Mean (SD) & Mean (SD) \\
\hline \multicolumn{11}{|l|}{ Visual foragers } \\
\hline Pluvialis dominica & & & & & & & $0.1(0.29)$ & $0.1(0.29)$ & & \\
\hline Pluvialis squatarola & & & & & & & $0.1(0.29)$ & & & \\
\hline Charadrius semipalmatus & & & & & & & $0.2(0.58)$ & $0.1(0.29)$ & & \\
\hline Arenaria interpres & & & $0.1(0.32)$ & & & & & & & \\
\hline Calidris alba & $0.5(1.58)$ & & & & & $1.1(3.75)$ & $1.0(2.89)$ & $0.6(1.73)$ & & \\
\hline Calidris bairdii & $5.6(17.71)$ & $1.5(2.80)$ & $0.3(0.67)$ & & & $1.3(3.28)$ & $1.5(3.12)$ & $2.6(5.11)$ & $0.5(1.73)$ & \\
\hline Calidris melanotos & & & $0.1(0.32)$ & $1.0(3.16)$ & & & $0.3(0.62)$ & $1.0(2.00)$ & & \\
\hline Charadrius nivosus & & & & & & $0.1(0.29)$ & & & & \\
\hline Charadrius collaris & $0.3(0.48)$ & $1.5(3.24)$ & $0.6(1.07)$ & & $0.2(0.63)$ & $0.3(0.62)$ & $1.9(1.62)$ & $1.1(1.51)$ & & \\
\hline Charadrius modestus & $0.1(0.32)$ & $3.0(5.35)$ & $1.6(2.50)$ & & $0.1(0.32)$ & & & & & \\
\hline Vanellus chilensis & $1.8(1.48)$ & $1.3(1.42)$ & $1.7(1.57)$ & $2.7(1.57)$ & $0.6(0.97)$ & $3.5(2.97)$ & $4.1(6.73)$ & $2.3(3.62)$ & $2.2(2.12)$ & $0.7(0.98)$ \\
\hline \multicolumn{11}{|l|}{ Tactile foragers } \\
\hline Himantopus mexicanus & $2.9(3.57)$ & $2.9(5.40)$ & $5.5(7.31)$ & $1.3(3.47)$ & $5.8(7.16)$ & $1.7(1.50)$ & $2.1(2.07)$ & $2.5(2.11)$ & $2.5(3.80)$ & $1.8(1.82)$ \\
\hline Haematopus palliatus & & $0.1(0.32)$ & $1.2(2.10)$ & & & $0.4(1.00)$ & $2.8(5.37)$ & $0.7(0.89)$ & & \\
\hline Numenius phaeopus & & $2.3(3.16)$ & $2.5(5.84)$ & $0.3(0.95)$ & $0.1(0.32)$ & $1.0(1.71)$ & $1.7(1.87)$ & $1.9(1.88)$ & $0.8(0.97)$ & $0.1(0.29)$ \\
\hline Limosa haemastica & & & & & & & & $0.2(0.39)$ & & \\
\hline Tringa semipalmata & & & $0.3(0.95)$ & & $0.2(0.63)$ & & & & & \\
\hline Tringa melanoleuca & $0.1(0.32)$ & $0.4(1.26)$ & $0.3(0.48)$ & $0.1(0.32)$ & $0.5(1.58)$ & $0.2(0.58)$ & $0.1(0.29)$ & $1.3(1.66)$ & $0.7(1.50)$ & $1.3(3.45)$ \\
\hline Tringa flavipes & & $0.2(0.63)$ & $0.6(0.70)$ & & $0.2(0.63)$ & & $0.1(0.29)$ & $0.4(0.67)$ & $0.8(1.22)$ & \\
\hline
\end{tabular}

SD: standard deviation 
Table 2. Abundance (mean \pm SD) of benthic orcenismsfound bysand flat areaduringaustral winter and springseasons. PS: Playa Sur; PN: Playa Norte; BN: Brazo Norte; TU: Totoral La ISla; TS Totoral Sur/ Abundancia (media \pm DE) de organismos bentónicos encontrados por planicie costera durante las estaciones de invierno y primavera. PS: Playa Sur; PN: Playa Norte; BN: Brazo Norte; TLl: Totoral La Isla; TS: Totoral Sur

\begin{tabular}{|c|c|c|c|c|c|c|c|c|c|c|}
\hline & \multicolumn{5}{|c|}{ AUSTRAL WINTER } & \multicolumn{5}{|c|}{ AUSTRAL SPRING } \\
\hline & PS & PN & $\mathrm{BN}$ & TLI & TS & PS & PN & $\mathrm{BN}$ & TLI & TS \\
\hline & Mean (SD) & Mean (SD) & Mean (SD) & Mean (SD) & Mean (SD) & Mean (SD) & Mean (SD) & Mean (SD) & Mean (SD) & Mean (SD) \\
\hline \multicolumn{11}{|l|}{ Polychaeta } \\
\hline Nereididae & $1.2(1.09)$ & $2.4(3.91)$ & $7.9(3.69)$ & $5.2(4.12)$ & $0.4(1.01)$ & $19.1(18.18)$ & $18.2(14.90)$ & $31.7(20.83)$ & $20.6(15.39)$ & $4.7(6.65)$ \\
\hline Spionidae & $7.7(7.02)$ & $1.4(1.59)$ & $10.7(11.51)$ & $4.8(5.78)$ & $3.4(4.64)$ & $21.4(24.19)$ & $4.0(4.85)$ & $6.9(9.68)$ & $11.9(11.15)$ & $7.2(8.20)$ \\
\hline Syllidae & & & $0.1(0.33)$ & & $0.1(0.33)$ & & & & & \\
\hline Oligochaeta & $4.1(6.35)$ & $1.0(1.73)$ & $4.2(7.31)$ & $10.4(13.38)$ & $13.1(22.03)$ & $18.9(47.26)$ & $2.9(3.98)$ & $7.4(14.04)$ & $10.1(26.63)$ & $20.9(21.27)$ \\
\hline Nematoda & $32.2(23.33)$ & $19.9(28.32)$ & $53.6(23.73)$ & $54.7(92.48)$ & $2.6(3.09)$ & $104.3(78.59)$ & $94.0(95.80)$ & $144.4(181.84)$ & $177.1(158.12)$ & $28.9(51.49)$ \\
\hline Gastropoda & & & $0.7(0.71)$ & $0.1(0.33)$ & $0.3(0.50)$ & & $0.3(0.71)$ & $1.2(1.48)$ & $1.0(1.66$ & $2.7(5.07)$ \\
\hline \multicolumn{11}{|l|}{ Crustacea } \\
\hline Amphipoda undet. & & & $0.1(0.33)$ & & & & & & & \\
\hline Corophiidae & & & $0.1(0.33)$ & $0.1(0.33)$ & & & & $0.2(0.44)$ & & \\
\hline Orchestoidea tuberculata & & & & & & & & $0.1(0.33)$ & & \\
\hline Ostracoda & $0.9(1.36)$ & $0.6(1.13)$ & $17.4(16.60)$ & $1.7(2.92)$ & $1.1(2.67)$ & $16.8(43.40)$ & $8.4(9.80)$ & $110.7(122.88)$ & $3.0(5.00)$ & \\
\hline Copepoda 1 & $1.1(2.09)$ & $0.1(0.33)$ & $0.2(0.44)$ & $1.7(3.04)$ & $18.7(33.33)$ & $8.9(23.35)$ & & $0.2(0.44)$ & $0.9(2.67)$ & $63.1(68.51)$ \\
\hline Copepoda 2 & & & & & $0.1(0.33)$ & $0.4(0.53)$ & $0.2(0.44)$ & $0.2(0.44)$ & $0.1(0.33)$ & $0.2(0.44)$ \\
\hline Collembola & & & & $0.1(0.33)$ & $0.2(0.44)$ & $0.2(0.67)$ & & & $0.3(0.50)$ & $3.0(3.64)$ \\
\hline Granuloreticulosa & & & $0.1(0.33)$ & & & & & & & \\
\hline Tardigrada & $0.1(0.33)$ & & & & & & & & & \\
\hline \multicolumn{11}{|l|}{ Insecta } \\
\hline Chironomidae (L) & $0.1(0.33)$ & & $0.1(0.33)$ & $1.1(2.09)$ & $0.9(1.54)$ & $1.9(4.01)$ & $0.6(0.88)$ & $1.6(3.32)$ & $1.8(4.97)$ & $3.7(5.02)$ \\
\hline Brachycera $1(\mathrm{~L})$ & & $0.1(0.33)$ & & & $0.2(0.67)$ & & & & $0.1(0.33)$ & $0.1(0.33)$ \\
\hline Brachycera $2(\mathrm{~L})$ & & $0.1(0.33)$ & & & & $0.3(0.71)$ & $0.1(0.33)$ & $1.2(3.67)$ & $0.1(0.33)$ & \\
\hline Dolichopodidae (L) & & & & & & & & & & $0.2(0.67)$ \\
\hline Diptera undet. & & & & & $0.2(0.67)$ & & & & & \\
\hline Coleoptera & & & & & $0.1(0.33)$ & & & & & $0.2(0.44)$ \\
\hline Lepidoptera (L) & & & & & $0.1(0.33)$ & & & & & \\
\hline Insecta undet.1 (L) & & & & & & & & & & $0.1(0.33)$ \\
\hline Insecta undet. $2(\mathrm{~L})$ & & & & & & & & & & $0.1(0.33)$ \\
\hline Insecta undet. $3(\mathrm{~L})$ & & & & & & & & & & $0.3(1.00)$ \\
\hline
\end{tabular}

(L): Larvae. SD: standard deviation 
Table 3. Results of Kruskal-Wallisstatistical test and post hoccontrasts using Mann-Whitney test with Bonferroni correctiontest comparingsediment grain size average, benthic imvertebrate abundance and foraging shorebird abundance among intertidal sand flats, during austral winter and spring seasons/ Resultados de la prueba estadística de Kruskal-Wallis y los contrastes a posteriori mediante la prueba de Mann-Whitney con la corrección de Bonferroni comparando promedio de tamaño de grano de sedimento, abundancia de invertebrados bentónicos y abundancia de aves playeras alimentándose entre planicies costeras, durante las estaciones de invierno y primavera

\begin{tabular}{|c|c|c|c|c|c|c|c|c|c|c|c|}
\hline \multirow{3}{*}{ Matrix } & \multirow{3}{*}{ Descriptor } & \multicolumn{5}{|c|}{ AUSTRAL WINTER } & \multicolumn{5}{|c|}{ AUSTRAL SPRING } \\
\hline & & \multicolumn{5}{|c|}{ Statistical comparisons between areas $(\alpha=0.05)$} & \multicolumn{5}{|c|}{ Statistical comparisons between areas $(\alpha=0.05)$} \\
\hline & & $\mathrm{H}(d f, \mathrm{n})$ & $P$-value & a posteriori test & $\mathrm{U}_{(2)}$ & $P$-value & $\mathrm{H}(d f, \mathrm{n})$ & $P$-value & a posteriori test & $\mathrm{U}_{(2)}$ & $P$-value \\
\hline \multirow[t]{2}{*}{ Sediments } & Average & $16.60(4 ; 45)$ & $<0.05^{*}$ & $\mathrm{PN} \neq \mathrm{TS}$ & 5.0 & $<0.05^{*}$ & $8.49(4 ; 45)$ & n.s. & & & \\
\hline & & & & $\mathrm{BN} \neq \mathrm{TS}$ & 7.0 & $<0.05^{*}$ & & & & & \\
\hline \multirow[t]{5}{*}{ Benthos } & Abundance & $21.05(4 ; 45)$ & $<0.05^{*}$ & $\mathrm{PN} \neq \mathrm{BN}$ & 0.5 & $<0.05^{*}$ & $11.71(4 ; 45)$ & $<0.05$ & $\mathrm{PN} \neq \mathrm{BN}$ & 11.0 & $<0.05$ \\
\hline & & & & $\mathrm{PN} \neq \mathrm{TLI}$ & 7.0 & $<0.05^{*}$ & & & $\mathrm{PN} \neq \mathrm{TS}$ & 11.5 & $<0.05$ \\
\hline & & & & $\mathrm{PN} \neq \mathrm{TS}$ & 8.0 & $<0.05^{*}$ & & & $\mathrm{TLI} \neq \mathrm{TS}$ & 17.5 & $<0.05$ \\
\hline & & & & $\mathrm{PN} \neq \mathrm{PS}$ & 10.5 & $<0.05^{*}$ & & & & & \\
\hline & & & & $\mathrm{BN} \neq \mathrm{PS}$ & 5.0 & $<0.05^{*}$ & & & & & \\
\hline \multirow[t]{4}{*}{ Shorebirds } & Abundance & $5.83(4 ; 50)$ & n.s. & & & & $15.67(4 ; 60)$ & $<0.05^{*}$ & $\mathrm{PN} \neq \mathrm{TLI}$ & 31.0 & $<0.05$ \\
\hline & & & & & & & & & $\mathrm{PN} \neq \mathrm{TS}$ & 13.5 & $<0.05^{*}$ \\
\hline & & & & & & & & & $\mathrm{BN} \neq \mathrm{TS}$ & 23.5 & $<0.05^{*}$ \\
\hline & & & & & & & & & $\mathrm{TS} \neq \mathrm{PS}$ & 34.0 & $<0.05$ \\
\hline
\end{tabular}

H: Kruskal-Wallis statistic; $d f$ : degree of freedom; a posteriori test $\mathrm{U}_{(2)}$ : Mann-Whitney test with Bonferroni correction; n.s.: not significant; *: $P$-value $<0.01$; PS: Playa Sur; PN: Playa Norte; BN: Brazo Norte; TLI: Totoral La Isla; TS: Totoral Sur 


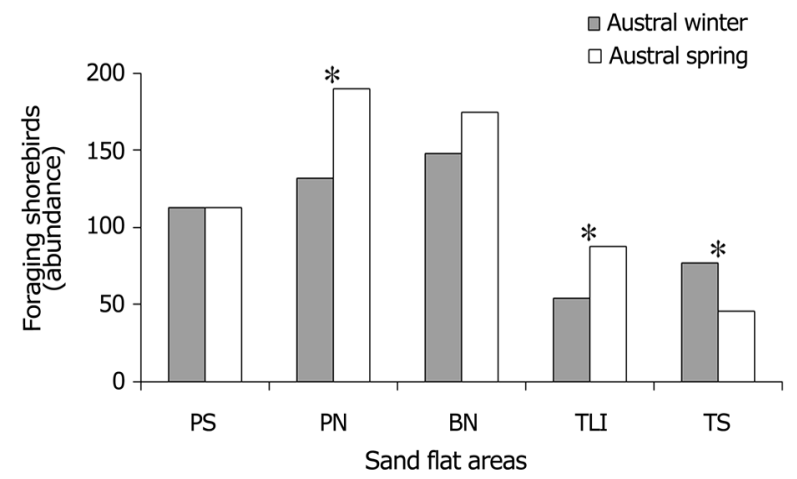

Figure 2. Total abundance of foraging shorebirds by sand flat areas during austral winter (gray column) and spring (white column) seasons. PS: Playa Sur; PN: Playa Norte; BN: Brazo Norte; TL: Totoral La Isla; TS: Totoral Sur. * indicates significant differences $(P<0.01) /$ Abundancia total de aves playeras alimentándose por planicie intermareal durante las estaciones de invierno (columna gris) y primavera (columna blanca). PS: Playa Sur; PN: Playa Norte; BN: Brazo Norte; TLI: Totoral La Isla; TS: Totoral Sur. ${ }^{*}$ indica diferencias significativas $(P<0,01)$

In terms of seasonal variability there was no difference among flats in shorebird abundance in winter. In contrast, the major difference among flats in shorebird abundance emerged during spring. PN (190 individuals) and TS (46 individuals) had significantlyhigher and lower shorebird abundances, respectively (Table 3). In addition a simple graphic view between winter and spring seasons had significant differences of total foraging shorebird abundance ratios (Yates' chi-square: $\mathrm{PN} \chi_{0.05 ; 1}^{2}=10.1$; $\operatorname{TLI} \chi_{0.05 ; 1}^{2}=7.7 ; \mathrm{TS} \chi_{0.05 ; 1}^{2}=7.3 ; P<0.01$ for all) (Fig. 2).

In austral spring, PS flat registered almost all species and TS flat only registered individuals of the more abundant species: black-necked stilt, southern lapwing, greater yellowlegs and whimbrel. Considering the whole wetland, our survey shows that neighborhood of tidal flats around the study site exhibit non-random spatial distributions of feeding shorebirds ( $s^{2}: x>>1$, for almost all species).

The shorebird ensemble showed highly significant variability among all flats in winter (ANOSIM, global $R=0.32, P=0.001$ ). Individual pairwise comparisons of flats (Table 4) revealed significant differences between TLI and PN-BN-TS flats. In spring, the shorebird ensemble showed highly significant variability among all flats (ANOSIM, global $R=0.162, P=$ 0.001 ). Pairwise comparisons (Table 4 ) revealed significant differences between TS and PN-BN flats.

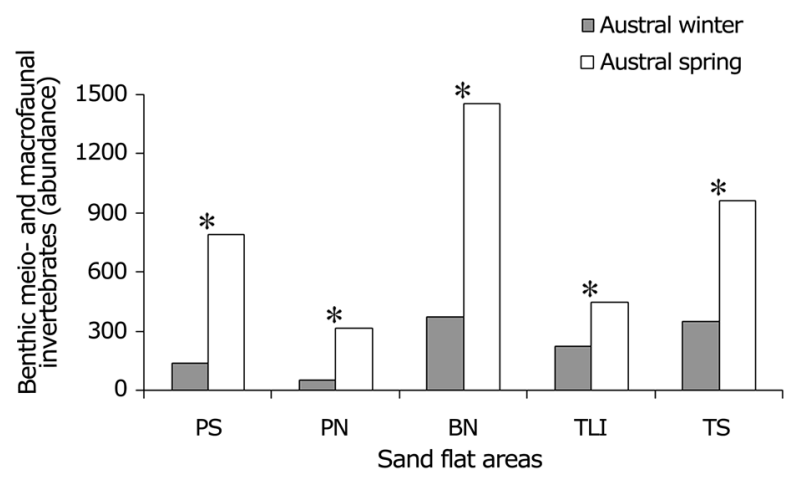

Figure 3. Total abundance of benthic macrofaunal taxa by sand flat areas during austral winter (gray column) and spring (white column) seasons. PS: Playa Sur; PN: Playa Norte; BN: Brazo Norte; TL: Totoral La Isla; TS: Totoral Sur. * indicates significant differences $(P<0.01)$ / Abundancia total de taxa macrobentónicos por planicie intermareal durante las estaciones de invierno (columna gris) y primavera (columna blanca). PS: Playa Sur; PN: Playa Norte; BN: Brazo Norte; TLI: Totoral La Isla; TS: Totoral Sur. * indica diferencias significativas $(P<0,01)$

\section{Benthos anALYsis}

The total meio- and macrobenthic invertebrates registered were 11,514 individuals, corresponding to 25 taxa (Table 2). Excluding nematodes, foraminiferans and tardigrads, almost all $(98 \%)$ invertebrates considered belonged to 4 major taxonomic groups ranging from 0.4 to $5.0 \mathrm{~cm}$ of total length: crustaceans $(45 \%)$, polychaetes $(34 \%)$, oligochaetes $(16 \%)$, and insects $(3 \%)$. Crustaceans consisted mostly of ostracods (62\%) and copepods (37\%), but also contained a few amphipods (1\%). Within the 3 families of polychaetes identified, $58.3 \%$ of individuals were nereidids, $41.6 \%$ were spionids, and $0.1 \%$ were syllids, being the most of them of small size. Oligochaetes were considered as a major taxonomic group. Insects were mainly represented by larvae of chironomids (75\%), and brachycers (16\%), but also contained a few minor groups (9\%: coleopters, dolichopodids, lepidopters, and others not identified).

The major difference among flats in benthic invertebrate abundance emerged from the analysis. PN (52 individuals) and PS (136 individuals) had significantly lower benthic invertebrate abundances than other flats in winter (Table 3), being PN the flat with markedly lower abundances in both seasons. BN (1,453 individuals) and TS (959 individuals) had significantly higher benthic invertebrate abundances than other flats in spring (Table 3 ). In addition a simple graphic view between winter and spring seasons had significant differences of total benthic invertebrate abundance ratios (Yates' chi-square: $\mathrm{PN} \chi_{0.05 ; 1}^{2}=185.2 ; \mathrm{BN} \chi_{0.05 ; 1}^{2}=636.1 ; \mathrm{TLI} \chi_{0.05 ; 1}^{2}=72.3$; $\mathrm{TS} \chi_{0.05 ; 1}^{2}=280.1 ; \mathrm{PS} \chi_{0.05 ; 1}^{2}=462.3 ; P<0.01$ for all) (Fig. 3). 
Table 4. Results of analysis of similarity (ANOSIM) and post hoc pairwise comparisons on sediment grain size composition, benthic invertebrate composition and foraging shorebird composition among intertidal sand flats, during austral winter and spring seasons / Resultados del análisis de similitud (ANOSIM) y las comparaciones a posteriori de a pares sobre la composición del tamaño de grano de los sedimentos, composición de invertebrados bentónicos y composición de aves playeras alimentándose entre planicies costeras, durante las estaciones de invierno y primavera

\begin{tabular}{|c|c|c|c|c|c|c|c|c|c|c|}
\hline \multirow{3}{*}{ Matrix } & \multicolumn{5}{|c|}{ AUSTRAL WINTER } & \multicolumn{5}{|c|}{ AUSTRAL SPRING } \\
\hline & \multicolumn{5}{|c|}{ ANOSIM between areas $(\alpha=0.05)$} & \multicolumn{5}{|c|}{ ANOSIM between areas $(\alpha=0.05)$} \\
\hline & $R$ global test & $P$-value & $R$ pairwis & ests & $P$-value & $R$ global test & $P$-value & $R$ pairwi & ests & $P$-value \\
\hline \multirow[t]{6}{*}{ Sediments } & 0.51 & $<0.05^{*}$ & PN - TLI & 0.47 & $<0.05^{*}$ & 0.46 & $<0.05^{*}$ & PN - TLI & 0.40 & $<0.05^{*}$ \\
\hline & & & $\mathrm{PN}$ - TS & 0.98 & $<0.05^{*}$ & & & PN - TS & 0.77 & $<0.05^{*}$ \\
\hline & & & $\mathrm{BN}-\mathrm{TS}$ & 0.75 & $<0.05^{*}$ & & & $\mathrm{BN}-\mathrm{TS}$ & 0.72 & $<0.05^{*}$ \\
\hline & & & TLI - TS & 0.56 & $<0.05^{*}$ & & & TLI - TS & 0.81 & $<0.05^{*}$ \\
\hline & & & TLI - PS & 0.36 & $<0.05^{*}$ & & & TLI - PS & 0.47 & $<0.05^{*}$ \\
\hline & & & TS - PS & 0.96 & $<0.05^{*}$ & & & TS - PS & 0.84 & $<0.05^{*}$ \\
\hline \multirow[t]{4}{*}{ Benthos } & 0.15 & $<0.05^{*}$ & $\mathrm{BN}-\mathrm{TS}$ & 0.53 & $<0.05^{*}$ & 0.24 & $<0.05^{*}$ & PN - TS & 0.64 & $<0.05^{*}$ \\
\hline & & & & & & & & $\mathrm{BN}-\mathrm{TS}$ & 0.71 & $<0.05^{*}$ \\
\hline & & & & & & & & TLI - TS & 0.41 & $<0.05^{*}$ \\
\hline & & & & & & & & TS - PS & 0.42 & $<0.05^{*}$ \\
\hline \multirow[t]{3}{*}{ Shorebirds } & 0.32 & $<0.05^{*}$ & PN - TLI & 0.60 & $<0.05^{*}$ & 0.16 & $<0.05^{*}$ & PN - TS & 0.34 & $<0.05^{*}$ \\
\hline & & & BN - TLI & 0.60 & $<0.05^{*}$ & & & BN - TS & 0.37 & $<0.05^{*}$ \\
\hline & & & TLI - TS & 0.70 & $<0.05^{*}$ & & & & & \\
\hline
\end{tabular}

*: $P$-value < 0.01; PS: Playa Sur; PN: Playa Norte; BN: Brazo Norte; TLI: Totoral La Isla; TS: Totoral Sur

The benthic macrofaunal communities showed highly significant variability among all flats in winter using abundances of major taxonomic groups (ANOSIM, global $R=0.146, P=$ 0.004). Individual pairwise comparisons of flats (Table 4) revealed significant differences between BN-TS flats. In spring, the benthic macrofaunal communities showed highly significant variability among all flats (ANOSIM, global $R=0.238, P=$ 0.001). Pairwise comparisons (Table 4) revealed significant differences between TS and all other flats.

\section{SEDiments anALYSIS}

The sediment sampled was classified as sand, ranging from fine to very fine sand. The majority of intertidal sand flat areas were dominated by fine sand (PN, BN, TLI, and PS). TS was dominated by very fine sand.

The major among-flat differences in grain-size group medians emerged during winter. The most clearly distinguishable flat from the others was TS, which had significantly the finest sediments by far (phi units > 3.3). There were no among-flat differences in grain-size group medians during spring (Table 3 ).

Results of analysis of similarity conducted on complete grain size distributions of each tidal flat revealed a significant difference among the flats (winter: ANOSIM, global $R=0.511, P=0.001$; spring: ANOSIM, global $R=0.462, P=0.001)$. Pairwise comparisons (Table 4) revealed significant differences between TLI-TS and PN-PS flats in both seasons.

\section{INTEGRATED ANALYSIS}

Global BEST match analysis between active matrices (i.e., sediments and benthos), composite active matrix and shorebirds fixed matrix were not significant in winter. On the other hand, when all 3 composited datasets were used concurrently (correlating sediment size composition and benthic macrofaunal composition with shorebird ensemble patterns), the BEST analysis optimized at $\rho_{s}=0.99(P=0.007)$ with 5 variables (Insecta, Oligochaeta, silt/clay, fine sand and very fine sand) in spring (Table 5).

Chi-square contingency table analysis between foraging shorebird species, and benthic trophic items, associated with particular tidal flat area were significant during both seasons (winter: foraging shorebird species $\chi_{0.05: 48}^{2}=469.6$; benthic trophic items $\chi_{0.05 ; 32}^{2}=247.3$; spring: foraging shorebird species $\chi_{0.05 ; 56}^{2}=272.5$; benthic trophic items $\chi_{0.05 ; 28}^{2}=1,114.2$ ). 
Table 5. Results of Global BEST analysis on shorebird fixed matrix using sediment, benthos and composite (sediment and benthos taken together) active matrices, during austral winter and spring seasons / Resultados del análisis BEST sobre la matriz fija aves playeras usando las matrices activas sedimento, bentos y compuesta (sedimento y bentos en conjunto), durante las estaciones de invierno y primavera

\begin{tabular}{|c|c|c|c|c|c|c|c|}
\hline \multirow{3}{*}{$\begin{array}{l}\text { Fixed } \\
\text { matrix }\end{array}$} & \multirow{3}{*}{ Active matrix } & \multirow{2}{*}{\multicolumn{3}{|c|}{$\begin{array}{c}\text { AUSTRAL WINTER } \\
\text { Global BEST match test } \\
(\alpha=0.05)\end{array}$}} & \multirow{2}{*}{\multicolumn{3}{|c|}{$\begin{array}{c}\text { AUSTRAL SPRING } \\
\text { Global BEST match test } \\
(\alpha=0.05)\end{array}$}} \\
\hline & & & & & & & \\
\hline & & rho & $P$-value & Variables & rho & $P$-value & Variables \\
\hline Shorebirds & Sediments & 0.15 & n.s. & & 0.87 & n.s. & \\
\hline Shorebirds & Benthos & 0.09 & n.s. & & 0.43 & n.s. & \\
\hline \multirow[t]{5}{*}{ Shorebirds } & Composite & 0.25 & n.s. & & 0.99 & $<0.05^{*}$ & Insecta \\
\hline & & & & & & & Oligochaeta \\
\hline & & & & & & & Silt/clay \\
\hline & & & & & & & Fine sand \\
\hline & & & & & & & Very fine sand \\
\hline
\end{tabular}

*: $P$-value $<0.01$; rho: Spearman correlation coefficient; n.s.: not significant

\section{DISCUSSION}

Both the total abundance of feeding shorebirds as well as the species composition varied across flats mainly in spring due to omnipresence of the residents black-necked stilt, southern lapwing and migrants like whimbrel and greater yellowlegs, with the intermittent presence of american golden plover, blackbellied plover, semipalmated plover, sanderling, hudsonian godwit, ruddy turnstone and willet, related to their southward migration, and the rufous-chested dotterel returning to their southern breeding areas (Aparicio 2006).

Visual foraging shorebirds (i.e., plovers, and rufous-chested dotterel) were registered mainly in BN and PN flats. BN and PN were characterized by the presence of chironomids, high abundance of ostracods and also polychaetes, all of them preys that have been registered in studies of dietary composition of shorebirds conducted in South America (Schlatter \& Jaramillo 1983, Meerhoff et al. 2013, Isacch et al. 2005, MartínezCurci et al. 2015). The southern lapwing was registered in all flats, mainly in PS, BN and TLI where were abundant oligochaetes and insects that are part of their diet (Schlatter \& Jaramillo 1983, Gantz et al. 2009).

On the other side, tactile and visual foraging shorebirds (i.e., black-necked stilt, whimbrel, greater yellowlegs, lesser yellowlegs, hudsonian godwit and american oystercatcher) were seen mainly in BN, PN and TLI. These tidal flats showed high abundance of nereid polychaetes and oligochaetes, all of them registered as preys on the diet of these shorebirds (Skagen \& Oman 1996, Tuckwell \& Nol 1997, Velasquez \& Navarro 2003). In addition, BN flat showed high abundance of ostracods, also registered as preys on the diet of hudsonian godwit (Martínez-Curci et al. 2015). During this study the rufous-chested dotterel, collared plover and Baird's sandpiper were seen occasionally capturing polychaete worms in the water line, and hudsonian godwit, black-necked stilt, whimbrel, greater yellowlegs and american oystercatcher were seen on shallow water also capturing polychaete worms, the more abundant benthic invertebrates registered in all tidal flats.

Based on some earlier studies of feeding shorebirds (Colwell \& Landrum 1993, Yates et al. 1993), the strong relationship between benthic invertebrates as prey and feeding shorebirds as predators is predictable. Shorebirds exhibit a great dietary flexibility and combine specific prey selection and opportunism to select the most abundant prey locally available (Skagen \& Oman 1996, Davis \& Smith 2001). We suggest that polychaete and oligochaete worms abundance, and seasonal abundance of copepods, ostracods and insects, play an important role for the small numbers of shorebirds that use this place as stopover site in winter and spring season during their migration along the coast of central Chile.

Global BEST analysis demonstrated that the shorebird ensemble distribution correlated strongly with local variation in 
the benthic invertebrate community and on sediment grain-size distribution taken together, explaining a large portion of the pattern in shorebird foraging habitat use only in spring. It is plausible that many species respond to food availability within Aconcagua River wetland. Although statistical analysis argues mainly insects and oligochaetes explain the pattern of distribution of shorebirds we consider that other groups of benthic invertebrates with locally high abundance also support the food requirements of shorebirds (Davis \& Smith 2001). In addition, grain size distributions, in that case the smaller fractions of sediments, also contribute to explain the pattern of distribution of shorebirds due to its influence over benthic invertebrate prey availability and their distributions.

Chi-square contingency table analysis, applied separately upon foraging shorebird and benthic invertebrate matrices, showed that the patterns of distributions of benthic taxa and foraging shorebirds are related to particular flat areas in the study site, suggesting that shorebirds prefer particular flats areas based on availability of benthic invertebrates present in there. During winter PS was dominated by Baird's sandpiper and sanderling, and also it was characterized by high abundance of spionid polychaetes; PN tidal flat was dominated by rufouschested dotterel, collared plover and whimbrel, and also it was characterized by high abundance of nereidid polychaetes; BN was dominated by american oystercatcher and whimbrel, and also it was characterized by the presence of spionid and nereidid polychaetes, oligochaetes and high abundance of ostracods; TLI was dominated by pectoral sandpiper and southern lapwing, and also it was characterized by high abundance of oligochaetes; and TS was dominated by black-necked stilt, and also it was characterized by the presence of oligochaetes and high abundance of copepods. During spring PS was dominated by sanderling and southern lapwing, and also it was characterized by high abundance of oligochaetes, spionid polychaetes, nereidid polychaetes and ostracods; PN tidal flat was dominated by american oystercatcher and collared plover, and also it was characterized by high abundance of nereidid polychaetes; BN was dominated by pectoral sandpiper and Baird's sandpiper, and also it was characterized by the presence of spionid polychaetes and high abundance of ostracods; TLI was dominated by lesser yellowlegs and black-necked stilt, and also it was characterized by high abundance of nereidid and spionid polychaetes; and TS was dominated by greater yellowlegs and black-necked stilt, and also it was characterized by the presence of oligochaetes, collembolans and high abundance of copepods.

Many species of birds, particularly migratory birds, have enormous routes from their summer nesting grounds to their temperate grounds with stopovers in between. As we remark, in Chile the coastal environments are discontinuous and small in size, and the wetland of Aconcagua River is not the exception, playing an important role for birds as stopover and feeding site to replenish their energy during migration. The loss or degradation of habitat at any component of these wetland systems, including the beginning and the end, vastly increases the already hazardous journeys. These results have important implications for shorebird conservation considering the variation in spatial and temporal use of habitat by a large variety of resident and migrant shorebird populations. It also would be considered for future wetlands management to ensure that critical and sensitive feeding areas are adequately protected, mainly in relation to environmental changes and other causes of habitat loss and destruction linked with worldwide decline in many shorebird populations (Colwell 2010).

In conclusion, our results showed some differences between tidal flat areas based on abundance and composition of benthic trophic items in both seasons (mediated by sediment characteristics), and also some relationships between foraging shorebirds and trophic items in particular tidal flat areas.

\section{ACKNOWLEDGMENTS}

We thank M.Allende for her willingness to assist us in the Parque Ecológico La Isla, Concón, and R. Schlatter for all suggestions made on our manuscript. We also thank the three anonymous reviewers for their rigorous and detailed comments.

\section{LITERATURE CITED}

Aparicio A. 2006. Abundance and phenology of migratory nonbreeding shorebirds on exposed sandy beaches of south central Chile. Wader Study Group Bulletin 111: 60-63.

Baker MC \& AEM Baker. 1973. Niche relationship among six species of shorebirds. Animal Behaviour 55: 1659-1667.

Bibby CJ, ND Burgess, DA Hill \& S Mustoe. 2000. Bird census techniques, 302 pp. Academic Press, London.

Blott SJ \& K Pye. 2001. GRADISTAT: a grain size distribution and statistics package for the analysis of unconsolidated sediments. Earth Surface Processes and Landforms 26: 12371248.

Burger J, M Howe, D Hahn \& J Chase. 1977. Effects of tide cycles on habitat selection and habitat partitioning by migrating shorebirds. Auk 94: 743-758.

Butler RW, NC Davidson \& RIG Morrison. 2001. Globalscale shorebird distribution in relation to productivity of nearshore ocean waters. Waterbirds 24: 224-232.

Clarke KR \& RN Gorley. 2006. PRIMER v6: User Manual/ Tutorial, 192 pp. PRIMER-E, Plymouth.

Colwell MA. 1993. Shorebird community patterns in a seasonally dynamic estuary. Condor 95: 104-114. 
Colwell MA. 2010. Shorebird ecology, conservation, and management, $344 \mathrm{pp}$. University of California Press, London.

Colwell MA \& SL Landrum. 1993. Nonrandom shorebird distribution and fine-scale variation in prey abundance. Condor 95: 94-103.

Davis CA \& LM Smith. 2001. Foraging strategies and niche dynamics of coexisting shorebirds at stopover sites in the Southern Great Plains. The Auk 118(2): 484-495.

Estades CF, MA Vukasovic \& V López. 2009. Las aves acuáticas del río Itata. En: Parra O, JC Castilla, H Romero, R Quiñones \& A Camaño (eds). La cuenca hidrográfica del río Itata, pp. 213-227. Editorial Universitaria de Concepción, Concepción.

Finn PG, CP Catterall \& PV Driscoll. 2008. Prey versus substrate as determinants of habitat choice in a feeding shorebird. Estuarine, Coastal and Shelf Science 80: 381-390.

Gantz A, S Sade \& J Rau. 2009. Winter diet and feeding preferences of the southern lapwing (Vanellus chilensis, Molina 1782) in pastures of southern Chile. Boletín Chileno de Ornitología 15(2): 87-93.

Ge B-M, Y-X Bao \& H-Y Cheng. 2011. Tempo-spatial variation of macrobenthic communities on a tidal flat of Wenzhou Bay, China. Revista de Biología Marina y Oceanografía 46(2): 281-286.

González AL, MA Vukasovic \& CF Estades. 2011. Variación temporal de la abundancia y diversidad de aves en el humedal del río Itata, región del Bío-Bío, Chile. Gayana 75(2): 170181.

Hammer Ø, DAT Harper \& PD Ryan. 2001. PAST: Paleontological statistics software package for education and data analysis. Palaeontologia Electronica 4(1): 1-9.

Isacch JP, CA Darrieu \& MM Martínez 2005. Food abundance and dietary relationships among migratory shorebirds using grasslands during the non-breeding season. Waterbirds 28(2): 238-245.

Jaramillo E, S Mulsow \& R Navarro. 1985. Intertidal and subtidal macroinfauna in the Queule River Estuary, South of Chile. Revista Chilena de Historia Natural 58: 127-137.

Johnson JA, BA Andres, HP Sitters, J Valenzuela, LJ Niles, AD Dey, MK Peck \& LA Espinosa. 2007. Counts and captures of Hudsonian Godwits and Whimbrels on Chiloé Island, Chile, January-February 2007. Wader Study Group Bulletin 113: 47-52.

Kraan C, J Van der Meer, A Dekinga \& T Piersma. 2009. Patchiness of macrobenthic invertebrates in homogenized intertidal habitats: hidden spatial structure at a landscape scale. Marine Ecology Progress Series 383: 211-224.

Marquet PA, S Abades \& I Barría. 2012. Distribución y conservación de humedales costeros: una perspectiva geográfica. En: Fariña JM \& A Camaño (eds). Humedales costeros de Chile. Aportes científicos a su gestión sustentable, pp. 1-19. Ediciones Universidad Católica de Chile, Santiago.
Martínez C \& C Cortez. 2007. Características hidrológicas y sedimentológicas en el estuario del río Aconcagua, Chile Central. Revista de Geografía Norte Grande 37: 63-74.

Meerhoff EI, LR Rodríguez-Gallego \& S Claramunt. 2013. Dieta de ocho especies de aves costeras en la barra de laguna de Rocha, Uruguay. Boletín de la Sociedad Zoológica del Uruguay 22(1): 12-22.

Morrison RIG \& JP Myers. 1987. Wader migration systems in the New World. Wader Study Group Bulletin 49 Suppl. IWRB Special Publication 7: 57-69.

Morrison RIG \& RK Ross. 1989. Atlas of Nearctic shorebirds on the coast of South America, 325 pp. Canadian Wildlife Service Special Publication, Environment Canada, Ottawa.

Myers JP, RIG Morrison, PZ Antas, BA Harrington, TE Lovejoy, M Sallaberry, SE Senner \& A Tarak. 1987. Conservation strategy for migratory species. American Scientist 75: 19-26.

Piersma T. 1987. Hop, skip, or jump? Constraints on migration of arctic waders by feeding, fattening, and flight speed. Limosa 60: 185-194.

Quijón P \& E Jaramillo. 1993. Temporal variability in the intertidal macroinfauna in the Queule River Estuary, Southcentral Chile. Estuarine, Coastal and Shelf Science 37: 655667.

Quinn DJ \& DJ Hamilton. 2012. Variation in diet of Semipalmated Sandpipers (Calidris pusilla) during stopover in the upper Bay of Fundy, Canada. Canadian Journal of Zoology 90: 1181-1190.

Richter W. 1985. Distribution of the soft-bottom macroinfauna in an estuary of Southern Chile. Marine Biology 86:93-100.

Sallaberry M, E Tabilo, C Kleese \& J Abarca. 1996. The Chilean shorebirds network (RECAP). International Wader Studies 8: 71-78.

Schlatter R \& W Sielfeld. 2006. Avifauna y mamíferos acuáticos de humedales en Chile. En: Vila I, A Veloso, R Schlatter \& C Ramírez (eds). Macrófitas y vertebrados de sistemas límnicos de Chile, pp. 141-179. Editorial Universitaria, Santiago.

Schlatter RP \& E Jaramillo. 1983. Ornitocenosis del litoral costero de Mehuín, Chile. El Hornero 12 (número extraordinario): 149-164.

Skagen SK. 2006. Migration stopovers and the conservation of Arctic-breeding calidridine sandpipers. Auk 123: 313-322.

Skagen SK \& HD Oman. 1996. Dietary flexibility of shorebirds in the Western Hemisphere. Canadian Field-Naturalist 110: 419-444.

Suazo CG, AM Arriagada \& JR Rau. 2012. Ephemeral aquatic bird assemblages in estuarine wetlands from Southcentral Chile: using an intertidal flat habitat during the austral summer. Estuarine and Coasts 35(4): 1137-1143. 
Tabilo E, R Jorge, R Riquelme, A Mondaca, C Labra, J Campusano, M Tabilo, M Varela, A Tapia \& M Sallaberry. 1996. Management and conservation of the habitats used by migratory shorebirds at Coquimbo, Chile. International Wader Studies 8: 79-84.

Torres P, J Ortiz, S Figueroa, R Jerez \& D Arcos. 2012. Caracterización del humedal costero Mataquito, Provincia de Curicó, Región del Maule. En: Fariña JM \& A Camaño (eds). Humedales costeros de Chile. Aportes científicos a su gestión sustentable, pp. 289-350. Ediciones Universidad Católica de Chile, Santiago.

Tuckwell J \& E Nol. 1997. Foraging behaviour of american oystercatchers in response to declining prey densities. Canadian Journal of Zoology 75: 170-185.

Udden JA. 1914. Mechanical composition of clastic sediments. Bulletin of the Geological Society of America 25: 655-744.

VanDusen BM, SR Fegley \& CH Peterson. 2012. Prey distribution, physical habitat features, and guild traits interact to produce contrasting shorebird assemblages among foraging patches. PLoS ONE 7(12): e52694. <doi:10.1371/ journal.pone.0052694>
Velásquez CR \& RA Navarro. 1993. The influence of water depth and sediment type on the foraging behavior of whimbrels. Journal of Field Ornithology 62(2): 149-157.

Warnock N. 2010. Stopping vs. staging: the difference between a hop and a jump. Journal of Avian Biology 41: 621-626.

Wentworth CK. 1922. A scale of grade and class terms for clastic sediments. Journal of Geology 30: 377-392.

Wilson HW. 1990. Relationship between prey abundance and foraging site selection by Semipalmated Sandpipers on a Bay of Fundy mudflat. Journal of Field Ornithology 61: 9-19.

Yates MG, JD Goss-Custard, S McGrorty, KH Lakhani, SEA Le V Dit Durell, RT Clarke, WE Rispin, L Moy, TJ Yates, RA Plant \& AJ Frost. 1993. Sediments characteristics, invertebrate densities and shorebird densities on the inner banks of the Wash. Journal of Applied Ecology 30: 599-614. 